\title{
Evaluation of the perceptual interaction among key aroma compounds in milk fan by gas chromatography-olfactometry, odor threshold, and sensory analyses
}

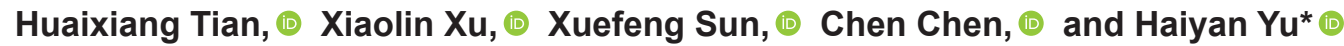 \\ Department of Food Science and Technology, Shanghai Institute of Technology, Shanghai 201418, China
}

\begin{abstract}
To evaluate the perceptual interactions among key aroma compounds found in milk fan, propanoic acid, butanoic acid, octanoic acid, octanal, nonanal, 2-nonanone, and ethyl hexanoate were analyzed by threshold values, aroma addition experiments (subthreshold), Feller's additive model (threshold), and $\sigma-\tau$ diagrams (threshold and superthreshold) at various concentrations. Aroma addition experiments highlighted that 7 key aroma compounds had significant effects on the aroma intensity of the sensory attributes of milk fan at the concentrations measured in a milk fan sample, and they might have synergistic effects with other compounds. The components of the 7 aroma compounds in binary mixtures showed synergistic effects from 21 binary mixtures by Feller's additive model. The components of 9 mixtures showed synergistic effects in the superadditive region $(\sigma>1.05)$, and 3 mixtures showed additive effects in the additive region $(0.95<\sigma<1.05)$ of the $\sigma-\tau$ diagrams for all 168 binary mixtures. The results showed that perceptual interactions among the key aroma compounds in milk fan vary with different concentrations and threshold ratios. These results are helpful for further understanding the aroma of milk fan and improving the quality of its aroma.
\end{abstract}

Key words: milk fan, key aroma compound, perceptual interaction, aroma addition experiment, Feller's additive model and $\sigma-\tau$ diagram

\section{INTRODUCTION}

Milk fan is a traditional Chinese cheese that has been produced for more than a thousand years and is a famous delicacy of the Bai ethnic minority in Yunnan Province (Liu et al., 2009). An aroma is an important characteristic attribute of food, and it can be used as an important indicator of monitoring food quality

Received November 9, 2019.

Accepted March 15, 2020.

*Corresponding author: hyyu@sit.edu.cn
(Togay et al., 2017; Hailu et al., 2018; Khattab et al., 2019). The key aroma compounds of foods can produce the perception of aromas in the human brain through their interaction with olfactory organs (Dunkel et al., 2014). Our previous research determined the key aroma compounds in Chinese milk fan cheese. Samples from 6 of the most popular manual workshops in Yunnan Province were analyzed via flavoromics approaches. The results identified propanoic acid, butanoic acid, octanoic acid, octanal, nonanal, 2-nonanone, and ethyl hexanoate as the key aroma compounds (Tian et al., 2019). The research on identification and the perceptual interaction of the key aroma compounds of milk fans are of great significance to promote the industrial production process of milk fans and effectively increase domestic cheese production.

The aroma components of many foods are complex, and it is recognized that the aroma is not simply equal to the sum of its odor-active compounds, and perceptual interactions, such as promoting and limiting functions, are indeed found for the aroma components (Cain and Drexler, 1974; Berglund and Olsson, 1993; Zhu et al., 2017). The perception interaction of complex odor mixtures makes it difficult to draw reasonable conclusions due to the complexity and diversity of the interactions between the different aroma components (Ferreira, 2012a; Wu et al., 2012; Niu et al., 2018). Aroma synergy generally means that the aroma perception intensity of the mixture is greater than the sum of the aroma perception intensity of each component in the mixture. That is, suppose that the A compound has an aroma intensity of $\mathrm{x}$ at the $\mathrm{n}$ concentration, $\mathrm{B}$ has an aroma intensity of $\mathrm{y}$ at the $\mathrm{n}$ concentration, and the $\mathrm{AB}$ mixture has an aroma intensity of $\mathrm{z}$ at a concentration of $2 \mathrm{n}$. When $\mathrm{z}>\mathrm{x}+\mathrm{y}$, synergy occurs (Lytra et al., 2013; Ferrer-Gallego et al., 2014; Cameleyre et al., 2015). At present, research methods for aroma synergy mainly include the threshold method, Feller's additive model, odor activity value determination, and the $\sigma-\tau$ diagram method (Ferreira, 2012a,b). These methods mainly determined the synergy between components through the changes of threshold, odor activity value, 
and aroma intensity before and after the combination of aroma components. Many studies have been done on the synergy of aroma compounds. Zhu et al. (2017) used a threshold method and aroma addition experiments combined with electronic nose technology to conduct a synergistic study of 24 aroma compounds in oolong tea. Their results showed that substances with similar structures or aromas might have synergistic or additive effects, whereas substances with different structures or aromas often have a masking effect. Lytra et al. (2014) used Feller's additive model to study the effect of dimethyl sulfide on the fruity aroma in complex mixtures containing 12 red wine esters. The results showed that the presence of dimethyl sulfide in the mixture significantly reduced the "olfactory threshold" of the fruit and increased its effect on the overall aroma intensity. Tempere et al. (2016) used Feller's additive model, sensory analysis, and GC-MS analysis to study the effects of ethyl phenols in wines (under low thresholds and superthresholds) on the perception of wine fruit aroma. The results showed that the perception of fruity aroma was masked and the aroma of the wine was affected when ethyl phenols were at low threshold and superthreshold concentrations. Cameleyre et al. (2015) used a combination of recombination, omission, and addition experiments and threshold olfactometry to study the effects of 5 higher alcohols on the wine aroma and used a $\sigma-\tau$ diagram to explore their aroma effects. Lytra et al. (2012) evaluated red wine via HPLC, obtained 25 aroma components, and used $\sigma-\tau$ diagrams to study the sensory interactions between the components. It was found that a component with a very weak fruit aroma had an additive effect on the aroma of fresh fruit, whereas a component imparting caramel and lactic acid aromas had a masking effect on the fresh fruit aroma and an additive effect on the berry aroma.

However, perceptual interactions among the aroma components in milk fan have not yet been determined. Therefore, the purpose of this research was to assess the perceptual interactions among key aroma compounds in milk fan by aroma addition experiments (subthreshold), Feller's additive model (threshold), and $\sigma$ - $\tau$ diagrams (threshold and superthreshold) at various concentrations. Exploring the perceptual interactions between substances is another key step in understanding the aroma of milk fan and will further help to improve the quality of its aroma.

\section{MATERIALS AND METHODS}

\section{Chemicals}

Propionic acid, butanoic acid, pentanoic acid, hexanoic acid, heptanoic acid, octanoic acid, n-decanoic acid, hexanal, octanal, nonanal, (E)-2-octenal, pentanol, hexanol, 1-octen-3-ol, nonanol, paracresol, 2-heptanone, 3-octanone, 2-nononone, acetophenone, ethyl butyrate, ethyl caproate, and D-limonene were purchased from Sigma-Aldrich (St. Louis, MO). These chemical substances were detected to be the odor-active compounds of milk fan Y6 sample in our previous study (Tian et al., 2019). All of the chemicals were of chromatographic grade.

\section{Sensory Evaluation}

General Conditions. Sensory analyses were performed as described by Martin and de Revel (1999). Samples were evaluated at controlled room temperature $\left(20^{\circ} \mathrm{C}\right)$ in individual booths using covered, brown, odorless glass bottles, containing $10 \mathrm{~mL}$ of liquid and coded with 3-digit random numbers. This process lasted 5 min.

Sensory Panel. The sensory evaluations were performed by 15 panelists ( 8 males and 7 females with an average age of $23 \mathrm{yr}$ ). All panelists belonged to the School of Perfume and Aroma Technology, Shanghai Institute of Technology, and had previously received professional training. However, they were not informed about the aim of the experiment. They were selected from 40 candidates based on their experience in evaluating cheese and their performance in 3-alternative forced-choice (3-AFC) tests. They attended 5 sessions per week for 8 wk. This sensory panel participated in all the experiments involving sensory evaluation in this study. The specific sensory evaluation experiment was designed with reference to the experimental contents of the following different parts.

\section{Gas Chromatography-Olfactometry Analysis of Reference Compounds}

Gas chromatography-olfactometry analysis was performed to ensure that the high-purity reference compounds did not contain any odorous impurities. Reference compounds were identified using an Agilent 7890 GC system in conjunction with an olfactory detection port (Gerstel ODP-2, Mulheim and der Ruhr, Germany). Reference compounds were separated with an HP-Innowax $(60 \mathrm{~m} \times 0.25 \mathrm{~mm} \times 0.25 \mu \mathrm{m}$; (Agilent Technologies, Santa Clara, CA) fused quartz capillary column. The experimental procedures were the same as in our previous study (Tian et al., 2019). Five panelists were randomly selected from the above-mentioned sensory panelists for this research. Each sample was analyzed in triplicate. The GC-olfactometry analysis found that none of the reference compounds purchased were contaminated with any pungent odor. After flame 
ionization detector analysis, the results showed that the chemical substances used had no obvious impurities. The substances purity was higher, which did not have other effects on the results of the subsequent series experiment.

\section{Aroma Addition Experiments}

The key aroma compounds (propanoic acid, butanoic acid, octanoic acid, octanal, nonanal, 2-nonanone, and ethyl hexanoate) were added to the aroma reconstitution (AR) at the concentrations measured in sample Y6 of our previous study (Tian et al., 2019). Aroma reconstitution was the AR model without the 7 key aroma compounds from sample Y6. The aroma addition experiments were performed by the sensory panelists, who were the same as the above-mentioned sensory panelists. The odor descriptors (fruity, cheesy, milky, sour, and fatty) were chosen in preliminary tests by the panelists (Majcher et al., 2018), and they were the 5 most representative aroma descriptors for the aroma of milk fan, according to previous sensory studies. The 5 descriptors were defined as follows, and relevant material objects or chemical standards were used as references as outlined in our previous studies (Aday and Yuceer, 2014; Tian et al., 2019). The 4-mL mixture of the key aroma compounds and $\mathrm{AR}$ or $\mathrm{AR}$ alone were kept in an odor-proof container $(5 \mathrm{~mL})$ until the panelists' evaluation. The odor properties were rated from 0 (none) to 10 (very strong). The tests were performed in triplicate.

\section{Measurement of Odor Threshold}

The odor threshold was measured based on ASTM (ASTM Standard, 2011) and previous studies (Saison et al., 2009). Fifteen panelists participated in this study, who were the same as the above-mentioned sensory panelists. The panelists were first exposed to familiarity with the standard solution and were informed of the nature of the additive. A series of test samples were prepared by adding a fresh milk solution matrix and adding the test substances to brown glass bottles. The panelists conducted several 3-AFC (ISO 13301, 2002) tests with sample concentrations ranging from high to low with a 2 -fold interval until they could not recognize the aroma. If the panelist was able to correctly recognize the aroma in both trials of the same sample, the same test was continued for samples of the next lowest concentration. The best-estimate threshold was the geometric mean of the final unrecognized concentration and the adjacent higher concentration (Guadagni et al., 1963).

\section{Discriminative Testing Methods (Feller's Additive Model)}

Each session included 10 replicates of 3-AFC experiments. Each experiment had one positive sample. The estimated threshold (Table 1) of the compound premeasured by 15 panelists (who were the same with the above-mentioned sensory panelists) was the intermediate concentration, diluted or increased by a factor of 2 , and 10 suitable sample concentrations were selected to determine the linear range of the fitted curve (AR diluted in a $50-\mathrm{mL}$ matrix by $0.1 / 0.2 / 0.4 / 0.8 / 1.6 / 3.1 /$ $6.3 / 12.5 / 25 / 50)$. The olfactory threshold is defined as the concentration with a detection probability of $50 \%$. The concentration/response function is a psychometric function and conforms to the sigmoid curve $[y=1$ / $\left.\left(1+\mathrm{e}^{(-\lambda \mathrm{x})}\right)\right]$, where $\mathrm{y}$ is the detection probability, $\mathrm{x}$ is the logarithmic concentration of aroma compounds, e is Napier's constant, and lambda is a coefficient associated with the steepness of a function. The evaluation of the mixture interaction used Feller's additive model (Feller, 1968), as developed by Miyazawa et al. (2008). Sigma Plot 8 (Systat Software Inc., Erkrath, Germany) software was used for graphics processing and rendering and ANOVA transformation for nonlinear regression (Tempere et al., 2011, 2012). The detection probability $p(A B)$ of the mixture was defined as follows: $p(A B)=$ $p(A)+p(B)-p(A) p(B)$, where $p(A)$ represents the detection probability of component $A$ and $p(B)$ represents the detection probability of component B.

\section{Discriminative Testing Methods (б-т Diagrams)}

The $\sigma-\tau$ diagrams were plotted from the intensity of the 4 concentration gradients of the 2 compounds in each combination. The estimated threshold (Table 1) was taken as the initial concentration. The choice of the 4 concentrations of each substance was based on a suitable constant factor such that the 2 were approximately equal in the corresponding perceived intensity. The concentrations of 7 pairs of binary mixtures for $\mathrm{A} 1-\mathrm{A} 4$ and $\mathrm{B} 1-\mathrm{B} 4$ in the $\sigma-\tau$ diagram are shown in Table 2, where A1-A4 represents 4 concentrations of compound A from low to high and B1-B4 represents 4 concentrations of compound B from low to high. These experiments were performed by the same sensory panel (15 panelists, 8 males and 7 females with an average age of $23 \mathrm{yr}$ ) as mentioned above. The odor intensity of the binary mixtures and the perceived odor intensity of each unmixed odorant were studied. The aroma intensity rating was performed using 1-butanol as a reference standard (Atanasova et al., 2004a). The aroma intensity was rated from 0 (none) to 5 (very strong). 
Table 1. Average concentration, threshold, and SD of the key aroma compounds detected in the milk fan sample Y6

\begin{tabular}{|c|c|c|c|c|}
\hline Aroma compound $^{1}$ & $\begin{array}{c}\text { Concentration } \\
(? / \mathrm{kg})\end{array}$ & SD & $\begin{array}{c}\text { Threshold } \\
(? / \mathrm{kg})\end{array}$ & SD \\
\hline Propanoic acid & 347 & 18.06 & 51,200 & 84.07 \\
\hline Butanoic acid & 7,030 & 215.20 & 7,500 & 24.64 \\
\hline Octanoic acid & 1,719 & 78.87 & 25,600 & 120.88 \\
\hline Octanal & 29 & 1.41 & 160 & 3.29 \\
\hline Nonanal & 198 & 4.48 & 1,600 & 29.41 \\
\hline 2-Nonanone & 244 & 27.97 & 52,000 & 116.04 \\
\hline Ethyl hexanoate & 1,001 & 50.93 & 1,024 & 34.28 \\
\hline
\end{tabular}

${ }^{1}$ Key aroma compounds detected in the milk fan sample Y6 in our previous study (Tian et al., 2019).

The experimental data were also reported in the graph of 2 parameters $[\sigma=f(\tau)]$ for the binary mixture (Patte and Laffort, 1979). In this function, $\tau$ is the ratio of the perceived intensity (I indicates the aroma intensity of aroma compounds) of unmixed $\mathrm{B}$ (or $\mathrm{A}$ ) in the mixture, $\tau_{\mathrm{A}}=\mathrm{I}_{\mathrm{A}} /\left(\mathrm{I}_{\mathrm{A}}+\mathrm{I}_{\mathrm{B}}\right)$ or $\tau_{\mathrm{B}}=\mathrm{I}_{\mathrm{B}} /\left(\mathrm{I}_{\mathrm{A}}+\mathrm{I}_{\mathrm{B}}\right)$, and $\sigma$ is the ratio between the perceived intensity of the mixture and the perceived intensity of its components: $\sigma=\mathrm{I}_{\text {mix }}$ $/\left(\mathrm{I}_{\mathrm{A}}+\mathrm{I}_{\mathrm{B}}\right)$, which reflects the level of interaction.

The location of the experimental points reflects the level of interaction. First, the intensity may be as strong as the sum of the perceived intensities of the unmixed components, at which point they are fully additive $(\sigma=1)$. When the intensity is stronger than the sum of its components, then superadditve effects occur $(\sigma>1)$; when the intensity is lower than the sum of its components, low additive effects occur $(\sigma<1)$. In addition, more detailed distinctions were made for the low additive effects: if the mass intensity of the mixture is greater than, medium, or less than the mass intensity of a single compound, it is defined as "partially additive," "compromise," or "subtractive," respectively (Frijters, 1987). For each sample, the significance of the perceived interaction observed by the test was statistically calculated by calculating a $95 \%$ confidence interval for the mean intensity of $\sigma$ and $\tau$ from the 15 subjects.

\section{Statistical Analysis}

Descriptive data and the differences between samples were analyzed by ANOVA and Duncan's test using SPSS v13.0 (SPSS Inc., Chicago, IL). The statistically significant level was 5\% $(P<0.05)$. Sigma Plot 12.0 (Systat Software Inc.) software was used to fit S-curves (Feller's additive model), and Origin 9.0 (OriginLab Corporation, Northampton, MA) was used to draw the $\sigma-\tau$ diagram.

\section{RESULTS AND DISCUSSION}

\section{Effect of Key Aroma Compounds on the Overall Aroma of Milk Fan}

The effect of the key aroma compounds on the overall aroma of milk fan was investigated by adding the key aroma compounds (propanoic acid, butanoic acid, octanoic acid, octanal, nonanal, 2-nonanone, and ethyl hexanoate) to the AR at the concentrations measured

Table 2. The concentrations of 7 pairs of binary mixtures for A1-A4 and B1-B4 in the $\sigma-\tau$ diagram (threshold and superthreshold) ${ }^{1}$

\begin{tabular}{lll}
\hline Binary mixture & Aroma compound & Concentration $(? / \mathrm{kg})$ \\
\hline (a) Nonanal and octanoic acid & Nonanal (A1-A4) & $1,024 / 2,048 / 4,096 / 8,192$ \\
& Octanoic acid (B1-B4) & $25,600 / 51,200 / 102,400 / 204,800$ \\
(b) Nonanal and propanoic acid & Nonanal (A1-A4) & $160 / 320 / 640 / 1,280$ \\
& Propanoic acid (B1-B4) & $51,200 / 204,800 / 409,600 / 819,200$ \\
(c) Ethyl hexanoate and 2-nonanone & Ethyl hexanoate (A1-A4) & $1,024 / 2,048 / 4,096 / 8,192$ \\
& 2-Nonanone (B1-B4) & $12,800 / 25,600 / 51,200 / 102,400$ \\
(d) Ethyl hexanoate and octanal & Ethyl hexanoate (A1-A4) & $1,024 / 2,048 / 4,096 / 8,192$ \\
& Octanal (B1-B4) & $160 / 320 / 640 / 1,280$ \\
(e) Ethyl hexanoate and butanoic acid & Ethyl hexanoate (A1-A4) & $1,024 / 2,048 / 4,096 / 8,192$ \\
& Butanoic acid (B1-B4) & $1,600 / 3,200 / 6,400 / 12,800$ \\
(f) 2-Nonanone and propanoic acid & 2-Nonanone (A1-A4) & $51,200 / 102,400 / 204,800 / 409,600$ \\
& Propanoic acid (B1-B4) & $51,200 / 102,400 / 204,800 / 409,600$ \\
(g) Octanal and butanoic acid & Octanal (A1-A4) & $160 / 320 / 640 / 1,280$ \\
& Butanoic acid (B1-B4) & $1,600 / 3,200 / 6,400 / 12,800$ \\
\hline
\end{tabular}

${ }^{1} \mathrm{~A} 1-\mathrm{A} 4$ represents 4 concentrations of compound A from low to high, and B1-B4 represents 4 concentrations of compound B from low to high. 
in sample Y6. Table 1 shows that the concentrations of the key aroma compounds in sample Y6 were less than the measured thresholds (i.e., they were at subthreshold levels). For the threshold, a certain difference was observed between the reference value in the literature and the measured value. This may be due to changes in olfactory thresholds depending on the matrix, experimental conditions, and sensory evaluators (Lytra et al., 2015). When the compounds were added to the solution, the intensity of the descriptors changed. Figure 1 shows that the addition of 7 key aroma compounds could highlight the intensity of the "fruity" descriptor to varying degrees, and compared with that of the AR, the intensity ratio ranged from 1.18 to 1.53 after the addition of the key aroma compounds. The possible cause of this situation is that the addition of these key aroma compounds had a significant interaction with compounds with "fruity" descriptors, such as some aldehydes (Xiao and Zhu, 2018) and esters (Lytra et al., 2013). Our previous study (Tian et al., 2019) found that ethyl hexanoate contributed to the fruity aroma of milk fan. Figure 1 shows that the addition of propanoic acid, octanoic acid, and 2-nonanone could highlight the intensity of the "cheesy" descriptor to varying degrees, and compared with that of the AR, the intensity ratios were $1.05,1.17$, and 1.25 , respectively. In our previous study (Tian et al., 2019) found that octanoic acid and 2-nonanone contributed to the cheesy aroma of milk fan. Moreover, the addition of butanoic acid reduced the "cheesy" intensity to within the range of 0.13 . The possible reason for this was that the addition of butanoic acid exhibited a suppressive effect on the volatiles with a cheesy aroma in the solution. As a result, the intensity of the cheese flavor was reduced. The addition of octanoic acid and octanal could highlight the intensity of the "milky" descriptor to varying degrees, and compared with that of the AR, the intensity ratio was approximately 1.35 . The addition of propanoic acid and octanoic acid could highlight the intensity of the "sour" descriptor to varying degrees, and compared with that of the AR, the intensity ratio ranged from 1.09 to 1.14 . The reason for this may be that the sour aroma of propanoic acid and octanoic acid enhanced the intensity of the overall "sour" aroma or exhibited synergistic effects with other compounds. The addition of octanal and nonanal could highlight the intensity of the "fatty" descriptor to varying degrees, and compared with that of the AR, the intensity ratio ranged from 1.15 to 1.2. The reason for this may be that there was a synergistic effect between octanal and nonanal and other compounds. This was consistent with the conclusion of Zhu et al. (2017), which showed that the grass aroma of tea liquor increased significantly after adding (E)-2-hexenal at a subthreshold level.
In short, when these key aroma compounds were present at the concentrations observed in milk fan samples, they had significant effects on the aroma intensity of the sensory attributes of the milk fan. These key aroma compounds enhanced the intensity of sensory attributes to a certain extent. Additionally, they may have synergistic effects with other substances. The synergistic effects between these aroma compounds were the focus of our study and were further investigated in subsequent experiments. This also indicated that the subthreshold or perithreshold levels of certain odorants had an effect on the intensity of the odor consistent with that of the superthreshold (Lytra et al., 2013). For example, one study found that subthreshold wood components enhanced the fruity odor (Niu et al., 2018).

\section{Synergistic Effect in the Combination of Key Aroma Compounds by Feller's Additive Model}

To better understand the synergistic effect between the key aroma compounds of the milk fan, binary mixtures with threshold concentration ratios were studied. The detection proportion of each compound was calculated by Feller's additive model of each binary mixture (Cometto-Muñiz and Abraham, 2016). The 7 key aroma compounds produced 21 binary mixtures, each of which was mixed at the measured threshold ratio. As shown in the S-curves of Figure 2, in the (a) nonanal and octanoic acid, (b) nonanal and propanoic acid, (c) ethyl hexanoate and 2-nonanone, (d) ethyl hexanoate and octanal, (e) ethyl hexanoate and butanoic acid, (f) 2-nonanone and propanoic acid, and (g) octanal and butanoic acid pairs, the measured binary mixture thresholds were below the calculated thresholds by Feller's additive model when the detection probability was $50 \%$. The ratios of the calculated threshold to the measured threshold were 1.38, 1.12, 5.07, 3.45, 4.59, 1.03 , and 1.17, respectively, for the above-mentioned pairs. A possible reason for these phenomena was the synergy between binary mixtures. It is worth noting that these mixtures had 4 different structures. These data were inconsistent with previous studies (Atanasova et al., 2004b; Saison et al., 2009). Previous studies have found that compounds with similar structures and aromas may tend to exhibit synergistic or additive effects, and almost no interaction between compounds with different structure and aroma (Saison et al., 2009; Xiao and Zhu, 2018). However, the reasons for these results are unclear and require further study. The composition of the mixture has a great effect on the perception of volatile molecules (Lytra et al., 2013). The molecular size of the aroma compound, type of functional group, and magnitude of its volatility may be factors that affect its effect on the overall perception. In addition, the 
interaction between molecules may be affected by van der Waals forces, hydrogen bonding (Sáenz-Navajas et al., 2010; Lorrain et al., 2013), and the degree of tortuosity of the food matrix (Silva et al., 2013, 2015). Moreover, it may also be influenced by sensory panelists in different backgrounds (Ramírez-Rivera et al., 2017, (a)

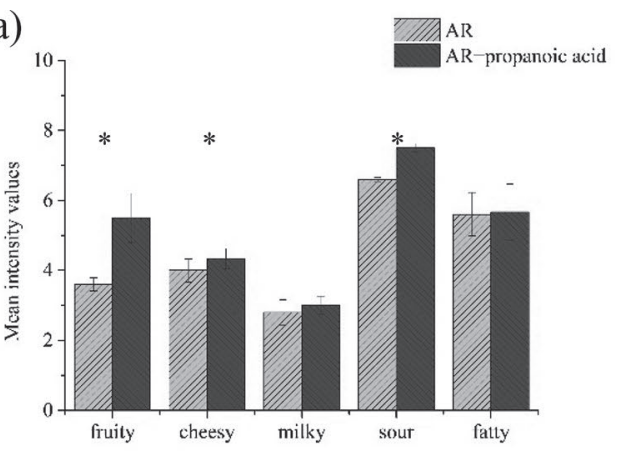

(c)

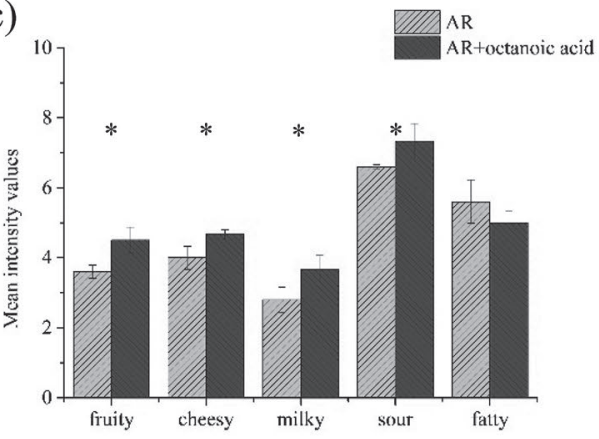

(e)

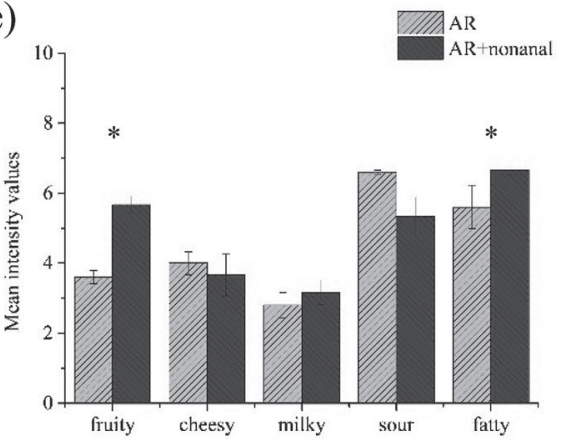

(g)

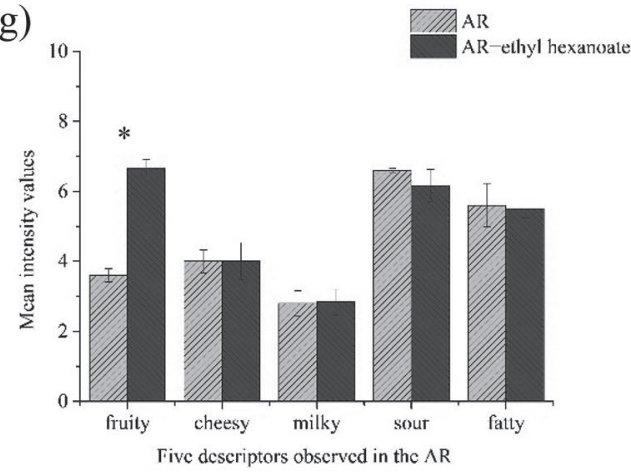

(b)

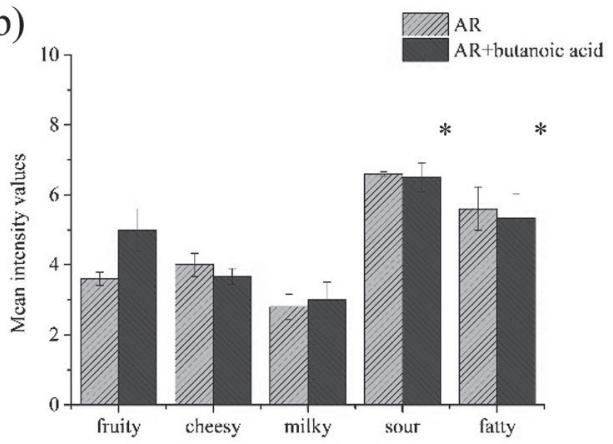

(d)

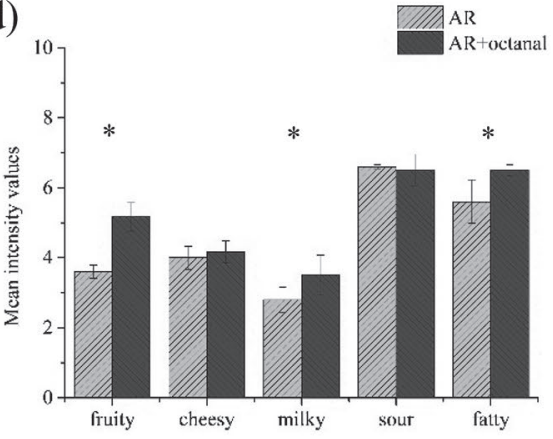

(f)

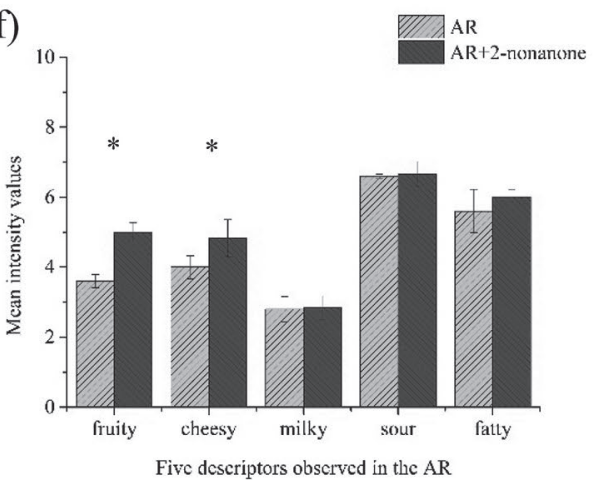

Figure 1. Effect of adding (a) propanoic acid, (b) butanoic acid, (c) octanoic acid, (d) octanal, (e) nonanal, (f) 2-nonanone, and (g) ethyl hexanoate to the aroma reconstitution (AR) to study the effect on overall aroma of milk fan. *Significant difference in the data between AR and AR with key aroma compounds added using Duncan's multiple comparison tests $(P<0.05)$. The error bars indicate the size of the SD of the data. 
$-\infty$ the curve of calculated

- the curve of measured

the fit curve of measured

----- the fit curve of measured

(a)

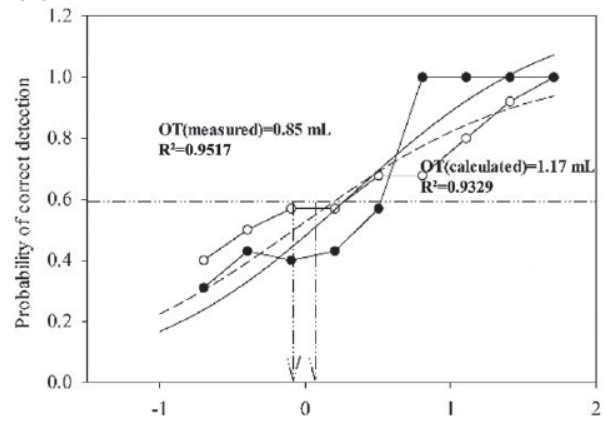

(c)

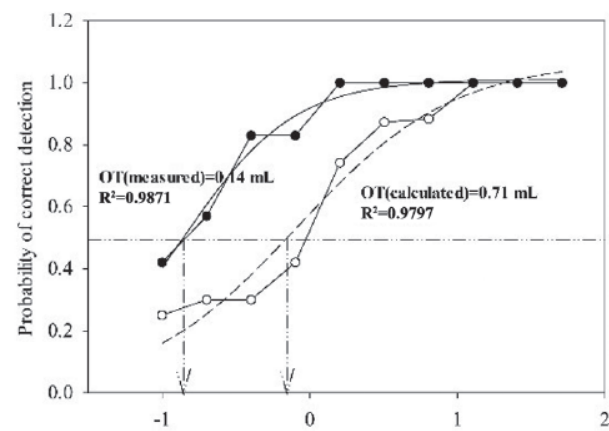

(e)

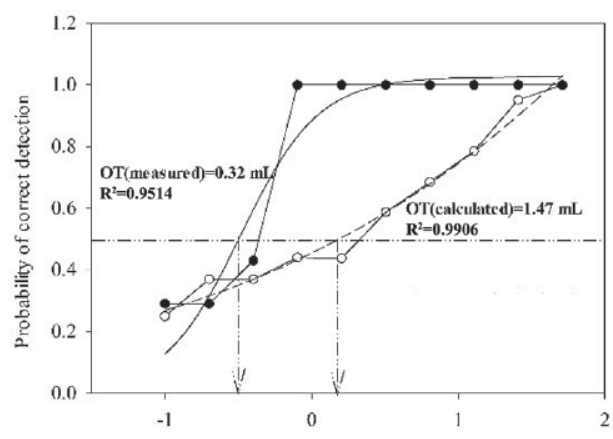

(g)

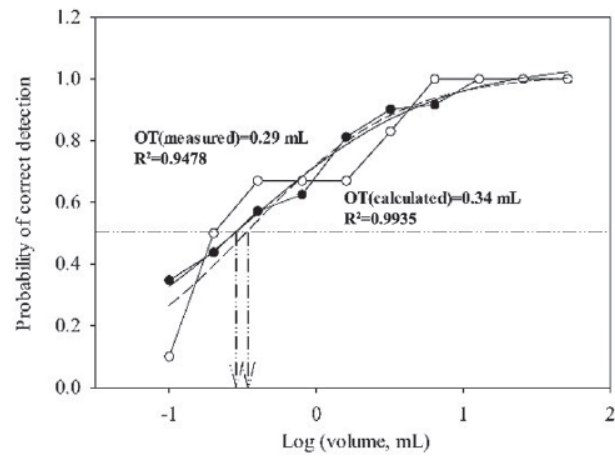

(b) nonanal-propanoic acid

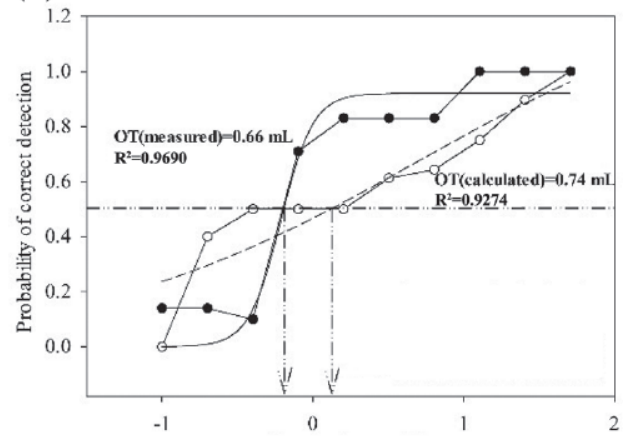

(d)

ethyl hexanoate + octanal

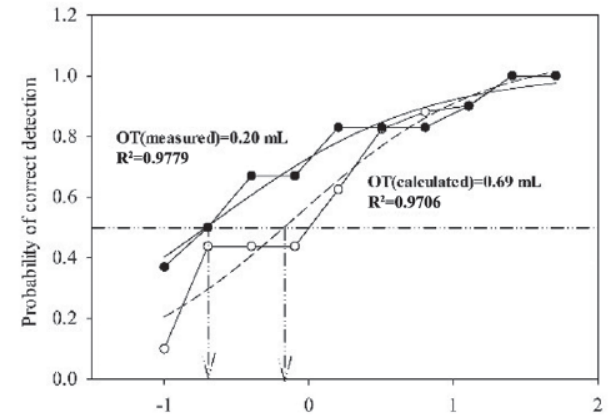

(f)

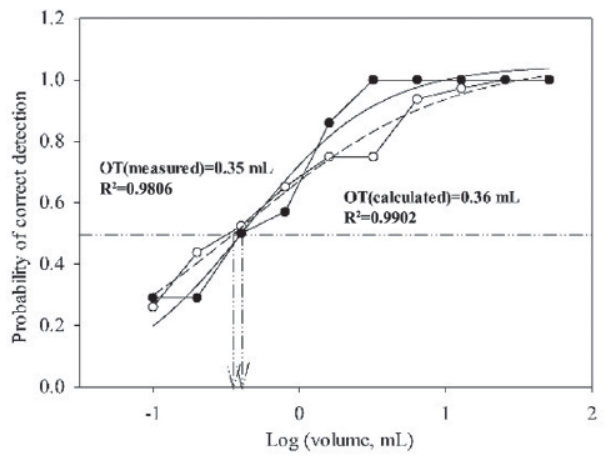

Figure 2. Perceptual interactions among 7 pairs of binary mixtures: (a) nonanal and octanoic acid, (b) nonanal and propanoic acid, (c) ethyl hexanoate and 2-nonanone, (d) ethyl hexanoate and octanal, (e) ethyl hexanoate and butanoic acid, (f) 2-nonanone and propanoic acid, and (g) octanal and butanoic acid. OT $=$ olfactory threshold. The curves are drawn according to S-curves. $\mathrm{R}^{2}=$ goodness of fit. 
(a)

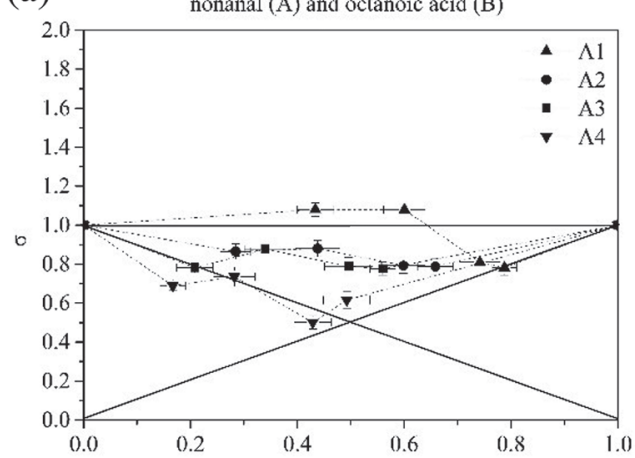

(b)

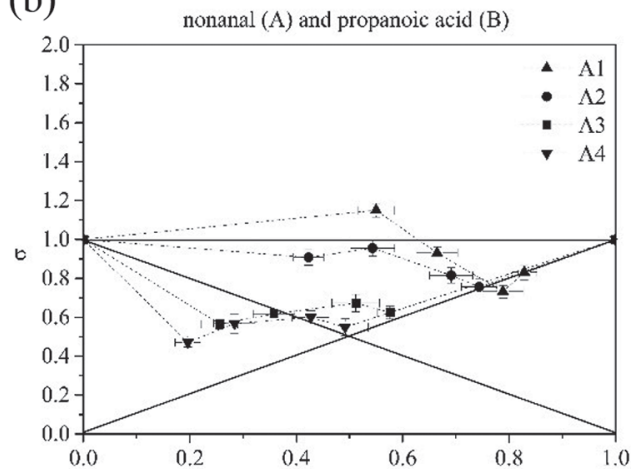

(c)

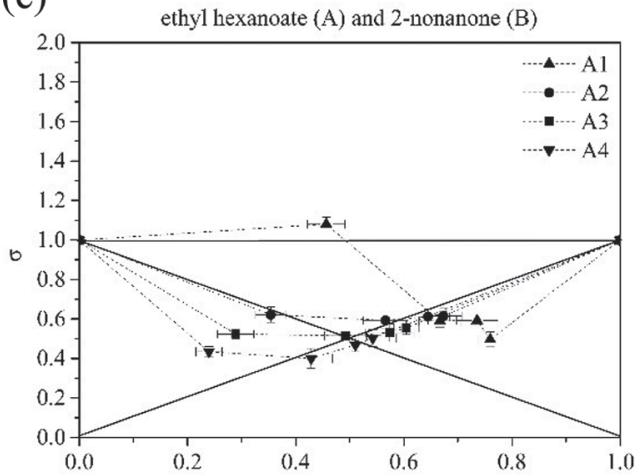

(d)

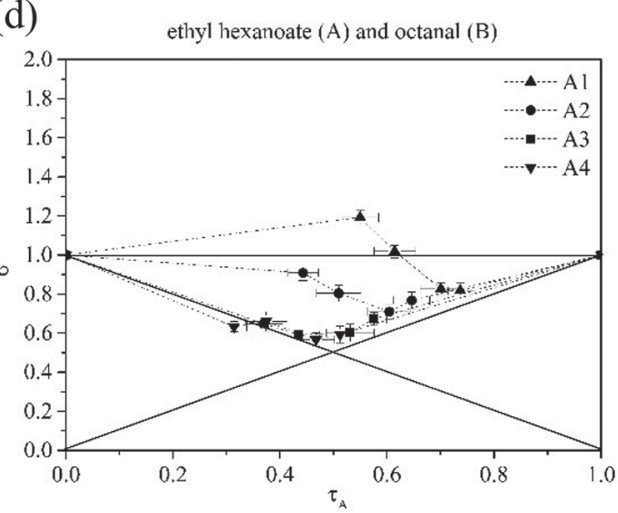

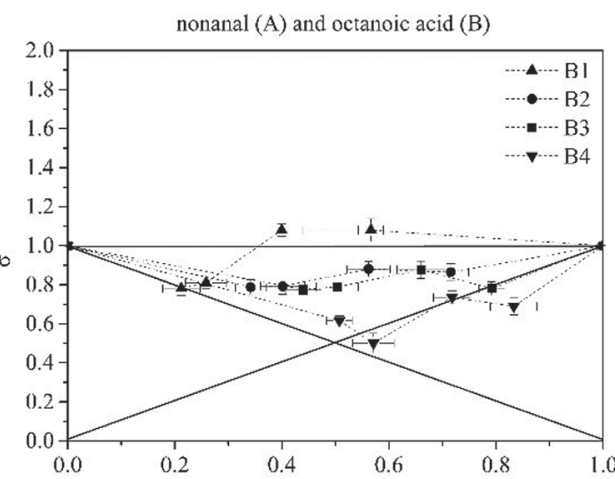
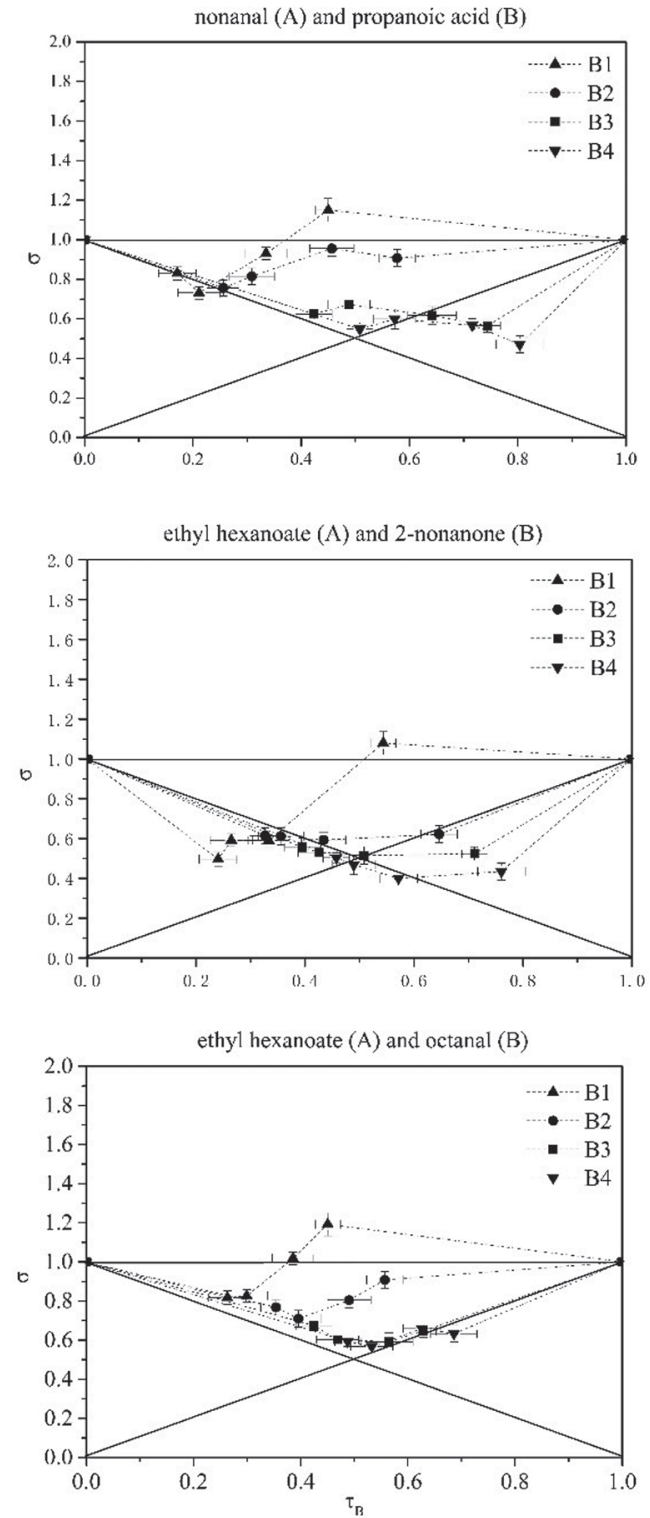

Figure 3. The $\sigma-\tau$ diagram (threshold and superthreshold) representations of 7 pairs of binary mixtures: (a) nonanal and octanoic acid, (b) nonanal and propanoic acid, (c) ethyl hexanoate and 2-nonanone, (d) ethyl hexanoate and octanal, (e) ethyl hexanoate and butanoic acid, (f) 2-nonanone and propanoic acid, and (g) octanal and butanoic acid. The error bars indicate the 95\% CI $\tau$ and $\sigma$ of the mean. A1-A4 represents 4 concentrations of compound A from low to high. B1-B4 represents 4 concentrations of compound B from low to high. 
2018). The study of these related factors will promote the development of food flavor at the industrial level and the production system level.

\section{Synergistic Effect in the Combination of Key Aroma Compounds by $\sigma-\tau$ Diagram}

Although the synergistic effects between the key aroma compounds of milk fan have been proven by Feller's additive model, they are only limited to the threshold concentration ratio, and synergistic effects between different concentration ratios are still unknown. Therefore, the $\sigma-\tau$ diagram method was used to study the perceptual interaction regarding the intensity of the aroma mixture. In previous work, 4 principles of odor intensity interaction received particular attention, including level independence, hypo-additivity, symmetry, and compromise (Cain and Drexler, 1974; Berglund and Olsson, 1993). As shown in Figure 3, the $\sigma=f(\tau)$ (Cameleyre et al., 2015) representation, depending on (e)

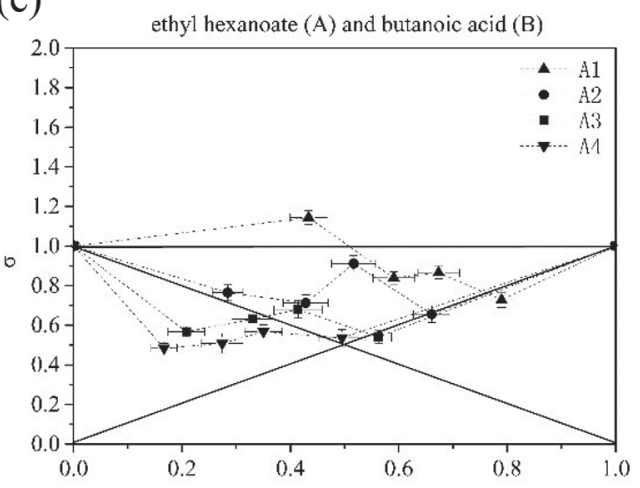

(f)

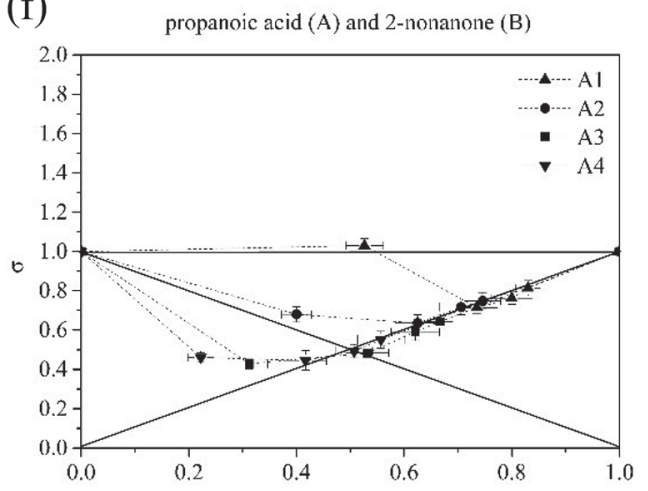

(g)

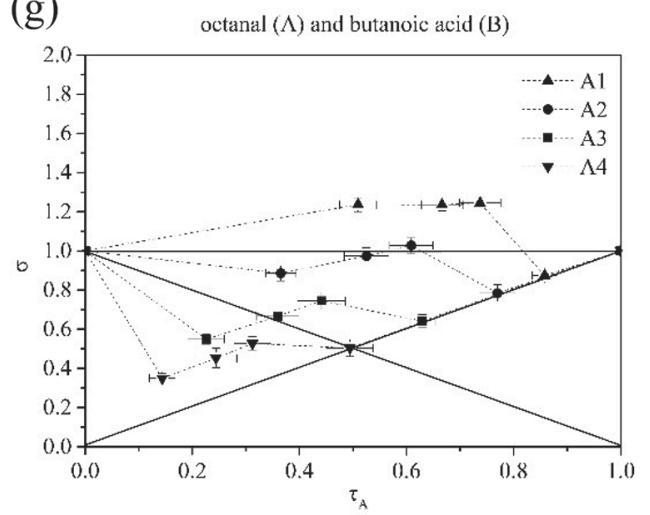

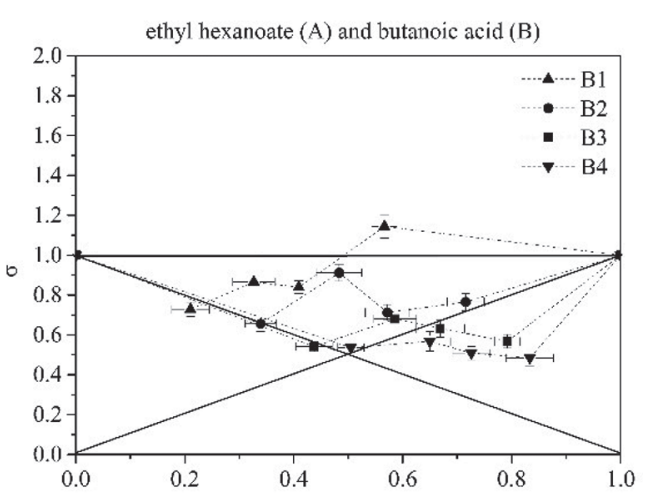
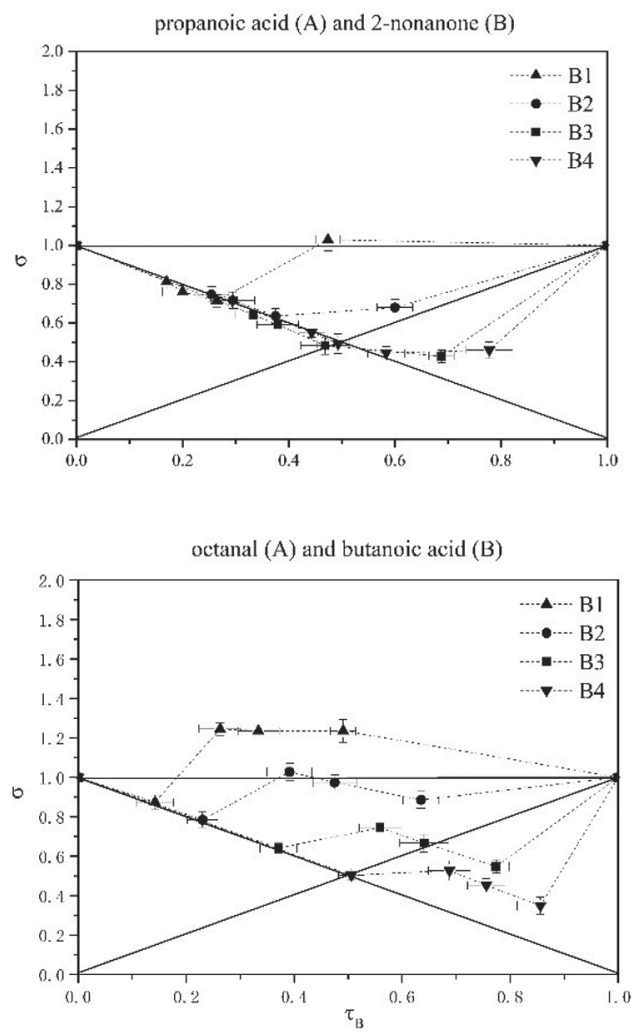

Figure 3 (Continued). The $\sigma-\tau$ diagram (threshold and superthreshold) representations of 7 pairs of binary mixtures: (a) nonanal and octanoic acid, (b) nonanal and propanoic acid, (c) ethyl hexanoate and 2-nonanone, (d) ethyl hexanoate and octanal, (e) ethyl hexanoate and butanoic acid, (f) 2-nonanone and propanoic acid, and (g) octanal and butanoic acid. The error bars indicate the $95 \%$ CI $\tau$ and $\sigma$ of the mean. A1-A4 represents 4 concentrations of compound A from low to high. B1-B4 represents 4 concentrations of compound B from low to high. 
the level of interaction, was divided into 5 different parts. Most data points for the binary mixtures were in the hypo-addition region. This meant that when the concentration ratio was in this range, the intensity of the mixture was weaker than the total intensity of the individual components but stronger than the intensity of the individual compounds. The hypo-additive effect was the most common, as the aroma intensity of the mixture was weaker than the sum of the component strengths. This phenomenon was common in binary mixtures and was consistent with previous research results (Patte and Laffort, 1979). For all 168 binary mixtures, only 9 mixtures were in the superadditive region $(\sigma>1.05)$. The compound concentration ratio was the measured threshold concentration ratio. Synergistic effects were found via $\sigma-\tau$ diagrams in the (a) nonanal and octanoic acid $(\tau=0.43, \sigma=1.08, \mathrm{~A} 1 \mathrm{~B} 1$; $\tau=0.60, \sigma=1.08, \mathrm{~A} 1 \mathrm{~B} 2$ ), (b) nonanal and propanoic acid $(\tau=0.55, \sigma=1.15$, A1B1 $),(\mathrm{c})$ ethyl hexanoate and 2-nonanone $(\tau=0.45, \sigma=1.08, \mathrm{~A} 1 \mathrm{~B} 1),(\mathrm{d})$ ethyl hexanoate and octanal $(\tau=0.54, \sigma=1.19$, A1B1), (e) ethyl hexanoate and butanoic acid $(\tau=0.43, \sigma=$ 1.14, A1B1), and (g) octanal and butanoic acid pairs ( $\tau$ $=0.51, \sigma=1.24, \mathrm{~A} 1 \mathrm{~B} 1 ; \tau=0.67, \sigma=1.24, \mathrm{~A} 1 \mathrm{~B} 2$; $\tau=0.74, \sigma=1.24$, A1B3). Close to the threshold concentration, synergistic effects easily occurred. This was consistent with the findings of $\mathrm{Wu}$ et al. (2012), who reported that enhancements would occur when the odor fineness introduced by the threshold odorant helps bring the overall odor of the mixture closer to the clearly defined odor objective. Binary mixtures with these concentration ratios were considered to have synergistic effects, which confirmed the conclusion of Feller's additive model. Figure 3 shows that 3 mixtures were in the so-called perfect additivity region $(0.95<\sigma<1.05)$ in the (d) ethyl hexanoate and octanal $(\tau=0.61, \sigma$ $=1.02, \mathrm{~A} 1 \mathrm{~B} 2)$, (f) 2-nonanone and propanoic acid $(\tau$ $=0.53, \sigma=1.03, \mathrm{~A} 1 \mathrm{~B} 1)$, and (g) octanal and butanoic acid pairs $(\tau=0.61, \sigma=1.03, \mathrm{~A} 2 \mathrm{~B} 3)$.

\section{CONCLUSIONS}

In this study, the threshold values of the key aroma compounds in milk fan, including propanoic acid, butanoic acid, octanoic acid, octanal, nonanal, 2-nonanone, and ethyl hexanoate, were determined. Synergistic effects among key aroma compounds in milk fan were evaluated by aroma addition experiments (subthreshold), Feller's additive model (threshold), and $\sigma-\tau$ diagrams (threshold and superthreshold) at various concentrations. These findings emphasize that perceptual interactions among key aroma compounds in milk fan vary with different concentrations and threshold ratios. Exploring the perceptual interactions between substances is another key step in understanding the aroma of milk fan and will further help to improve the quality of its aroma. It is very important to understand the aroma of milk fan and control the quality of its aroma in actual production.

\section{ACKNOWLEDGMENTS}

This work was sponsored by the National Natural Science Foundation of China (award no. 31771943) and the "Shu Guang" project (no. 16SG50) supported by Shanghai Municipal Education Commission and the Shanghai Education Development Foundation (Shanghai, China) for financial support. The authors have not stated any conflicts of interest.

\section{REFERENCES}

Aday, S., and Y. K. Yuceer. 2014. Physicochemical and sensory properties of Mihalic cheese. Int. J. Food Prop. 17:2207-2227. https:// doi.org/10.1080/10942912.2013.790904.

ASTM Standard. 2011. Standard practice for determination of odor and taste thresholds by a forced-choice ascending concentration series method of limits. E679-041-7.

Atanasova, B., D. Langlois, S. Nicklaus, C. Chabanet, and P. Etievant. 2004a. Evaluation of olfactory intensity: Comparative study of two methods. J. Sens. Stud. 19:307-326. https://doi.org/10.1111/ j.1745-459X.2004.tb00150.x.

Atanasova, B., T. D. Thomas-Danguin, D. Langlois, S. Nicklaus, and P. Etievant. 2004b. Perceptual interactions between fruity and woody notes of wine. Flavour Fragrance J. 19:476-482. https://doi .org/10.1002/ffj.1474.

Berglund, B., and M. J. Olsson. 1993. Odor-intensity interaction in binary and ternary mixtures. Percept. Psychophys. 53:475-482. https://doi.org/10.3758/BF03205195.

Cain, W. S., and M. Drexler. 1974. Scope and evaluation of odor counteraction and masking. Ann. N. Y. Acad. Sci. 237(1 Odors):427-439. https://doi.org/10.1111/j.1749-6632.1974.tb49876.x.

Cameleyre, M., G. Lytra, S. Tempere, and J. C. Barbe. 2015. Olfactory impact of higher alcohols on red wine fruity ester aroma expression in model solution. J. Agric. Food Chem. 63:9777-9788. https://doi.org/10.1021/acs.jafc.5b03489.

Cometto-Muñiz, J. E., and M. H. Abraham. 2016. Dose-response functions for the olfactory, nasal trigeminal, and ocular trigeminal detectability of airborne chemicals by humans. Chem. Senses 41:3-14. https://doi.org/10.1093/chemse/bjv060.

Dunkel, A., M. Steinhaus, M. Kotthoff, M. Nowak, B. Krautwurst, P. Schieberle, and T. Hofmann. 2014. Nature's chemical signatures in human olfaction: A foodborne perspective for future biotechnology. Angew. Chem. Int. Ed. Engl. 53:7124-7143. https://doi.org/ 10.1002/anie.201309508.

Feller, W. 1968. An Introduction to Probability Theory and Its Applications. Wiley Series in Probability and Mathematical Statistics, 3rd ed. Vol. 1. John Wiley and Sons, New York, NY.

Ferreira, V. 2012a. Revisiting psychophysical work on the quantitative and qualitative odour properties of simple odour mixtures: A flavour chemistry view. Part 1: Intensity and detectability. A review. Flavour Frag. J. 27:124-140.

Ferreira, V. 2012b. Revisiting psychophysical work on the quantitative and qualitative odour properties of simple odour mixtures: A flavour chemistry view. Part 2: qualitative aspects. A review. Flavour Fragrance J. 27:201-215. https://doi.org/10.1002/ffj.2091.

Ferrer-Gallego, G. R., J. M. Hernández-Hierro, J. C. Rivas-Gonzalo, and M. T. Escribano-Bailón. 2014. Sensory evaluation of bitterness and astringency sub-qualities of wine phenolic compounds: Syner- 
gistic effect and modulation by aromas. Food Res. Int. 62:11001107. https://doi.org/10.1016/j.foodres.2014.05.049.

Frijters, J. E. R. 1987. Psychophysical models for mixtures of tastants and mixtures of odorants. Ann. N. Y. Acad. Sci. 510:67-78. https: //doi.org/10.1111/j.1749-6632.1987.tb43469.x.

Guadagni, D. G., R. G. Buttery, S. Okano, and H. K. Burr. 1963. Additive effect of subthreshold concentrations of some organic compounds associated with food aromas. Nature 200:1288-1289. https: //doi.org/10.1038/2001288a0.

Hailu, Y., E. B. Hansen, E. Seifu, M. Eshetu, M. A. Petersen, R. Lametsch, F. Rattray, and R. Ipsen. 2018. Rheological and sensory properties and aroma compounds formed during ripening of soft brined cheese made from camel milk. Int. Dairy J. 81:122-130. https://doi.org/10.1016/j.idairyj.2018.01.007.

ISO 13301. 2002. Sensory Analysis-Methodology-General Guidance for Measuring Odour, Flavour and Test Detection Thresholds by a Three-Alternative Forced-Choice (3-AFC) Procedure. In Analyse Sensorielle. AFNOR, Paris, France.

Khattab, A. R., H. A. Guirguis, S. M. Tawfik, and M. A. Farag. 2019. Cheese ripening: A review on modern technologies towards flavor enhancement, process acceleration and improved quality assessment. Trends Food Sci. Technol. 88:343-360. https://doi.org/10 .1016/j.tifs.2019.03.009.

Liu, W., Z. H. Sun, J. C. Zhang, W. Gao, W. H. Wang, L. Wu, T. S. Sun, W. Chen, X. M. Liu, and H. P. Zhang. 2009. Analysis of microbial composition in acid whey for dairy fan making in Yunnan by conventional method and $16 \mathrm{~S}$ rRNA sequencing. Curr. Microbiol. 59:199-205. https://doi.org/10.1007/s00284-009-9423-x.

Lorrain, B., S. Tempere, N. Iturmendi, V. Moine, G. de Revel, and P. L. Teissedre. 2013. Influence of phenolic compounds on the sensorial perception and volatility of red wine esters in model solution: An insight at the molecular level. Food Chem. 140:76-82. https:// doi.org/10.1016/j.foodchem.2013.02.048.

Lytra, G., M. Cameleyre, S. Tempere, and J. C. Barbe. 2015. Distribution and organoleptic impact of ethyl 3-hydroxybutanoate enantiomers in wine. J. Agric. Food Chem. 63:10484-10491. https://doi .org/10.1021/acs.jafc.5b04332.

Lytra, G., S. Tempere, A. Le Floch. G. de Revel, and J.-C. Barbe. 2013. Study of sensory interactions among red wine fruity esters in a model solution. J. Agric. Food Chem. 61:8504-8513. https://doi .org/10.1021/jf4018405.

Lytra, G., S. Tempere, G. de Revel, and J. C. Barbe. 2012. Impact of perceptive interactions on red wine fruity aroma. J. Agric. Food Chem. 60:12260-12269. https://doi.org/10.1021/jf302918q.

Lytra, G., S. Tempere, S. Zhang, S. Marchand, G. D. Revel, and J. C. Barbe. 2014. Olfactory impact of dimethyl sulfide on red wine fruity esters aroma expression in model solution. J. Int. Sci. Vigne Vin 48:75-85. https://doi.org/10.20870/oeno-one.2014.48.1.1660.

Majcher, M. A., K. Myszka, A. Gracka, A. Grygier, and H. H. Jeleń 2018. Key odorants of Lazur-A Polish type mold-ripened cheese. J. Agric. Food Chem. 66:2443-2448. https://doi.org/10.1021/acs .jafc.6b04911.

Martin, N., and G. de Revel. 1999. Sensory evaluation: Scientific bases and oenological applications. J. Int. Sci. Vigne Vin 33:81-93.

Miyazawa, T., M. Gallagher, G. Preti, and P. Wise. 2008. Synergistic mixture interactions in detection of perithreshold odors by humans. Chem. Senses 33:363-369. https://doi.org/10.1093/chemse/ bjn004.

Niu, Y., Z. M. Yao, Z. Xiao, G. Zhu, J. Zhu, and J. Chen. 2018. Sensory evaluation of the synergism among ester odorants in light aroma-type liquor by odor threshold, aroma intensity and flash GC electronic nose. Food Res. Int. 113:102-114. https://doi.org/ 10.1016/j.foodres.2018.01.018.

Patte, F., and P. Laffort. 1979. An alternative model of olfactory quantitative interaction in binary mixtures. Chem. Senses 4:267-274. https://doi.org/10.1093/chemse/4.4.267.

Ramírez-Rivera, E. J., P. Díaz-Rivera, L. G. Ramón-Canul, J. M Juárez-Barrientos, J. Rodríguez-Miranda, E. Herman-Lara, W. Prinyawiwatkul, and J. A. Herrera-Corredor. 2018. Comparison of performance and quantitative descriptive analysis sensory profiling and its relationship to consumer liking between the artisanal cheese producers panel and the descriptive trained panel. J. Dairy Sci. 101:5851-5864. https://doi.org/10.3168/jds.2017-14213.

Ramírez-Rivera, E. J., L. G. Ramón-Canul, P. Díaz-Rivera, J. M. Juárez-Barrientos, E. Herman-Lara, W. Prinyawiwatkul, and J. A. Herrera-Corredor. 2017. Sensory profiles of artisan goat cheeses as influenced by the cultural context and the type of panel. Int. J. Food Sci. Technol. 52:1789-1800. https://doi.org/10.1111/ijfs .13452

Sáenz-Navajas, M. P., E. Campo, L. Culleré, P. Fernández-Zurbano, D. Valentin, and V. Ferreira. 2010. Effects of the nonvolatile matrix on the aroma perception of wine. J. Agric. Food Chem. 58:5574-5585. https://doi.org/10.1021/jf904377p.

Saison, D., D. P. De Schutter, B. Uyttenhove, F. Delvaux, and F. R. Delvaux. 2009. Contribution of staling compounds to the aged flavor of lager beer by studying their flavor thresholds. Food Chem. 114:1206-1215. https://doi.org/10.1016/j.foodchem.2008.10.078.

Silva, J. V. C., D. Legland, C. Cauty, I. Kolotuev, and J. Floury. 2015. Characterization of the microstructure of dairy systems using automated image analysis. Food Hydrocoll. 44:360-371. https://doi .org/10.1016/j.foodhyd.2014.09.028.

Silva, J. V. C., P. D. S. Peixoto, S. Lortal, and J. Floury. 2013. Transport phenomena in a model cheese: The influence of the charge and shape of solutes on diffusion. J. Dairy Sci. 96:6186-6198. https://doi.org/10.3168/jds.2013-6552.

Tempere, S., E. Cuzange, J. C. Bougeant, G. de Revel, and G. Sicard. 2012. Explicit sensory training improves the olfactory sensitivity of wine experts. Chemosens. Percept. 5:205-213. https://doi.org/ 10.1007/s12078-012-9120-1.

Tempere, S., E. Cuzange, J. Malak, J. C. Bougeant, G. D. Revel, and G. Sicard. 2011. The training level of experts influences their detection thresholds for key wine compounds. Chemosens. Percept 4:99-115. https://doi.org/10.1007/s12078-011-9090-8.

Tempere, S., M. H. Schaaper, E. Cuzange, R. de Lescar, G. de Revel, and G. Sicard. 2016. The olfactory masking effect of ethylphenols: Characterization and elucidation of its origin. Food Qual. Prefer. 50:135-144. https://doi.org/10.1016/j.foodqual.2016.02.004.

Tian, H. X., X. L. Xu, C. Chen, and H. Y. Yu. 2019. Flavoromics approach to identifying the key aroma compounds in traditional Chinese milk fan. J. Dairy Sci. 102:9639-9650. https://doi.org/10 .3168/jds.2019-16796.

Togay, S. O., O. Guneser, and Y. K. Yuceer. 2017. Evaluation of physicochemical, microbiological, sensory properties and aroma profiles of goat cheeses provided from Canakkale. Int. J. Dairy Technol. 70:514-525. https://doi.org/10.1111/1471-0307.12374.

Wu, K. N., B. K. Tan, J. D. Howard, D. B. Conley, and J. A. Gottfried. 2012. Olfactory input is critical for sustaining odor quality codes in human orbitofrontal cortex. Nat. Neurosci. 15:1313-1319. https://doi.org/10.1038/nn.3186.

Xiao, Z., and J. Zhu. 2018. Characterization of the major odor-active compounds in dry jujubes cultivars by application of gas chromatography-olfactometry and odor activity value. J. Agric. Food Chem. 66:7722-7734. https://doi.org/10.1021/acs.jafc.8b01366.

Zhu, J., F. Chen, L. Y. Wang, Y. W. Niu, and Z. B. Xiao. 2017. Evaluation of the synergism among volatile compounds in oolong tea infusion by odour threshold with sensory analysis and e-nose. Food Chem. 221:1484-1490. https://doi.org/10.1016/j.foodchem .2016.11.002.

\section{ORCIDS}

Huaixiang Tian @ https://orcid.org/0000-0002-6097-809X Xiaolin Xu ๑ https://orcid.org/0000-0001-7197-4521 Xuefeng Sun () https://orcid.org/0000-0003-0190-2412 Chen Chen ๑ https://orcid.org/0000-0002-4885-393X Haiyan Yu ๑ https://orcid.org/0000-0001-6472-1366 